\title{
On $\sigma$-type zero of Sheffer polynomials
}

\author{
Ajay K Shukla*, Shrinivas J Rapeli and Pratik V Shah
}

"Correspondence: ajayshukla2@rediffmail.com Department of Mathematics, S. V. National Institute of Technology, Surat, 395 007, India

\begin{abstract}
The main object of this paper is to investigate some properties of $\sigma$-type polynomials in one and two variables.

MSC: 33 C65; 33E99; 44A45; 46G25

Keywords: Appell sets; differential operator; Sheffer polynomials; generalized Sheffer polynomials
\end{abstract}

\section{Introduction}

In 1945, Thorne [1] obtained an interesting characterization of Appell polynomials by means of the Stieltjes integral. Srivastava and Manocha [2] discussed the Appell sets and polynomials. Dattoli et al. [3] studied the properties of the Sheffer polynomials. Recently Pintér and Srivastava [4] gave addition theorems for the Appell polynomials and the associated classes of polynomial expansions and some cases have also been discussed by Srivastava and Choi [5] in their book.

Appell sets may be defined by the following equivalent condition: $\left\{P_{n}(x)\right\}, n=0,1,2, \ldots$ is an Appell set [6-8] $\left(P_{n}\right.$ being of degree exactly $\left.n\right)$ if either

(i) $P_{n}^{\prime}(x)=P_{n-1}(x), n=0,1,2, \ldots$, or

(ii) there exists a formal power series $A(t)=\sum_{n=0}^{\infty} a_{n} t^{n}\left(a_{0} \neq 0\right)$ such that

$$
A(t) \exp (x t)=\sum_{n=0}^{\infty} P_{n}(x) t^{n}
$$

\section{Sheffer's A-type classification}

Let $\phi_{n}(x)$ be a simple set of polynomials and let $\phi_{n}(x)$ belong to the operator

$$
J(x, D)=\sum_{k=0}^{\infty} T_{k}(x) D^{k+1},
$$

with $T_{k}(x)$ of degree $\leq k$. If the maximum degree of the coefficients $T_{k}(x)$ is $m$, then the set $\phi_{n}(x)$ is of Sheffer A-type $m$. If the degree of $T_{k}(x)$ is unbounded as $k \rightarrow \infty$, we say that $\phi_{n}(x)$ is of Sheffer A-type $\infty$.

\section{Polynomials of Sheffer A-type zero}

Let $\phi_{n}(x)$ be of Sheffer A-type zero. Then $\phi_{n}(x)$ belong to the operator

$$
J(D)=\sum_{k=0}^{\infty} c_{k} D^{k+1},
$$

@ 2013 Shukla et al.; licensee Springer. This is an Open Access article distributed under the terms of the Creative Commons Attribution License (http://creativecommons.org/licenses/by/2.0), which permits unrestricted use, distribution, and reproduction in any medium, provided the original work is properly cited. 
in which $c_{k}$ are constants. Here $c_{0} \neq 0$ and $J \phi_{n}=\phi_{n-1}$. Furthermore, since $c_{k}$ are independent of $x$ for every $k$, a function $J(t)$ exists with the formal power series expansion

$$
J(t)=\sum_{k=0}^{\infty} c_{k} t^{k+1}, \quad c_{0} \neq 0 .
$$

Let $H(t)$ be the formal inverse of $J(t)$; that is,

$$
H(J(t))=J(H(t))=t
$$

Theorem (Rainville [9]) A necessary and sufficient condition that $\phi_{n}(x)$ be of Sheffer A-type zero is that $\phi_{n}(x)$ possess the generating function indicated in

$$
A(t) \exp (x H(t))=\sum_{n=0}^{\infty} \phi_{n}(x) t^{n}
$$

in which $H(t)$ and $A(t)$ have (formal) expansions

$$
H(t)=\sum_{n=0}^{\infty} h_{n} t^{n+1}, \quad h_{0} \neq 0, \quad A(t)=\sum_{n=0}^{\infty} a_{n} t^{n}, \quad a_{0} \neq 0 .
$$

Theorem (Al-Salam and Verma [10]) Let $\left\{P_{n}(x)\right\}$ be a polynomial set. In order for $\left\{P_{n}(x)\right\}$ to be a Sheffer A-type zero, it is necessary and sufficient that there exist (formal) power series

$$
H(t)=\sum_{j=1}^{\infty} h_{j} t^{j}, \quad h_{1} \neq 0, \quad A_{s}(t)=\sum_{j=0}^{\infty} a_{j}^{(s)} t^{j} \quad\left(\text { not all } a_{0}^{(s)} \text { are zero }\right)
$$

and

$$
\sum_{j=1}^{r} A_{j}(t) \exp \left(x H\left(\varepsilon_{j} t\right)\right)=\sum_{n=0}^{\infty} P_{n}(x) t^{n},
$$

where

$$
J(D) P_{n}(x)=P_{n-r}(x) \quad(n=r, r+1, \ldots) \text { where } J(D)=\sum_{k=0}^{\infty} a_{k} D^{k+r}, a_{0} \neq 0
$$

and $r$ is a fixed positive integer. The function $A(t)$ may be called the determining function for the $\operatorname{set}\left\{P_{n}(x)\right\}$.

Polynomial of $\sigma$-type zero $[9,11]$

Let $\left\{p_{n}(x)\right\}$ be a simple set of polynomials that belongs to the operator

$$
\begin{aligned}
& J(x, \sigma)=\sum_{k=0}^{\infty} T_{k}(x) \sigma^{k+1}, \\
& \sigma=D \prod_{i=1}^{q}\left(x D+b_{i}-1\right), \quad D=\frac{d}{d x}, \quad\left(J(x, \sigma) p_{n}(x)=p_{n-1}(x)\right),
\end{aligned}
$$


where $b_{i}$ are constants, not equal to zero or a negative integer, and $T_{k}(x)$ are polynomials of degree $\leq k$. We can say that this set is of $\sigma$-type $m$ if the maximum degree of $T_{k}(x)$ is $m, m=0,1,2, \ldots$.

A necessary and sufficient condition that $\phi_{n}(x)$ be of $\sigma$-type zero, with

$$
\sigma=D \prod_{i=1}^{q}\left(x D+b_{i}-1\right)
$$

is that $\phi_{n}(x)$ possess the generating function

$$
A(t)_{0} F_{q}\left(-; b_{1}, b_{2}, \ldots, b_{q} ; x H(t)\right)=\sum_{n=0}^{\infty} \phi_{n}(x) t^{n}
$$

in which $H(t)$ and $A(t)$ have (formal) expansions

$$
H(t)=\sum_{n=0}^{\infty} h_{n} t^{n+1}, \quad h_{0} \neq 0
$$

and

$$
A(t)=\sum_{n=0}^{\infty} a_{n} t^{n}, \quad a_{0} \neq 0
$$

Since $\phi_{n}(x)$ belongs to the operator $J(\sigma)=\sum_{k=0}^{\infty} c_{k} \sigma^{k+1}$, where $c_{k}$ are constant and $c_{0} \neq 0$.

\section{Main results}

Theorem 1 If $p_{n}(x)$ is a polynomial set, then $p_{n}(x)$ is of $\sigma$-type zero with $\sigma=D \prod_{m=1}^{q}(x D+$ $\left.b_{m}-1\right)$. It is necessary and sufficient condition that there exist formal power series

$$
H(t)=\sum_{n=0}^{\infty} h_{n} t^{n+1}, \quad h_{0} \neq 0
$$

and

$$
A_{i}(t)=\sum_{n=0}^{\infty} a_{n}^{(i)} t^{n} \quad\left(\text { not all } a_{0}^{(i)} \text { are zero }\right)
$$

such that

$$
\sum_{i=1}^{r} A_{i}(t)_{0} F_{q}\left(-; b_{1}, b_{2}, \ldots, b_{q} ; x H\left(\varepsilon_{i} t\right)\right)=\sum_{n=0}^{\infty} p_{n}(x) t^{n}
$$

where $\theta=x D$.

Proof Let $y_{i}={ }_{0} F_{q}\left(-; b_{1}, b_{2}, \ldots, b_{q} ; z_{i}\right)$, where $i=1,2, \ldots, r$, be a solution of the following differential equation:

$$
\left[\theta \prod_{m=1}^{q}\left(x D+b_{m}-1\right)-z_{i}\right] y_{i}=0, \quad \theta=x D, \quad D=\frac{d}{d x} \text {. }
$$


On substituting $z_{i}=x H\left(\varepsilon_{i} t\right)$ and keeping $t$ as a constant, where

$$
\sigma=D \prod_{m=1}^{q}\left(x D+b_{m}-1\right), \quad \theta=x D,
$$

we get

$$
\left[x D \prod_{m=1}^{q}\left(\theta+b_{m}-1\right)-x H\left(z_{i}\right)\right] y_{i}=0 .
$$

This can also be written as

$$
\sigma y_{i}=H\left(\varepsilon_{i} t\right) y_{i}
$$

or

$$
\sigma_{0} F_{q}\left(-; b_{1}, b_{2}, \ldots, b_{q} ; x H\left(\varepsilon_{i} t\right)\right)=H\left(\varepsilon_{i} t\right)_{0} F_{q}\left(-; b_{1}, b_{2}, \ldots, b_{q} ; x H\left(\varepsilon_{i} t\right)\right) .
$$

Operating $J(\sigma)$ on both sides of Equation (1) yields

$$
\begin{aligned}
J(\sigma) \sum_{n=0}^{\infty} p_{n}(x) t^{n} & =J(\sigma) \sum_{i=1}^{r} A_{i}(t)_{0} F_{q}\left(-; b_{1}, b_{2}, \ldots, b_{q} ; x H\left(\varepsilon_{i} t\right)\right) \\
& =\sum_{i=1}^{r} A_{i}(t) J\left(H\left(\varepsilon_{i} t\right)\right)_{0} F_{q}\left(-; b_{1}, b_{2}, \ldots, b_{q} ; x H\left(\varepsilon_{i} t\right)\right) \\
& =t \sum_{n=0}^{\infty} p_{n}(x) t^{n} \\
& =\sum_{n=1}^{\infty} p_{n-1}(x) t^{n} .
\end{aligned}
$$

Therefore, $J(\sigma) p_{0}(x)=0$ and $J(\sigma) p_{n}(x)=p_{n-1}(x), n \geq 1$.

Since $J(\sigma)$ is independent of $x$, using the definition of $\sigma$-type $[9,11]$, we arrive at the conclusion that $p_{n}(x)$ is $\sigma$-type zero.

Conversely, suppose $p_{n}(x)$ is of $\sigma$-type zero and belongs to the operator $J(\sigma)$. Now $q_{n}(x)$ is a simple set of polynomials, we can write

$$
\sum_{i=1}^{r}{ }_{0} F_{q}\left(-; b_{1}, b_{2}, \ldots, b_{q} ; x H\left(\varepsilon_{i} t\right)\right)=\sum_{n=0}^{\infty} p_{n}(x) t^{n}
$$

where $\varepsilon_{1}, \varepsilon_{2}, \ldots, \varepsilon_{r}$ are the roots of unity.

Since $q_{n}(x)$ is a simple set, there exists a sequence $c_{k}$ [10], independent of $n$, such that

$$
p_{n}(x)=\sum_{k=0}^{n} c_{n-k} q_{k}(x)
$$


and

$$
\sum_{n=0}^{\infty} p_{n}(x) t^{n}=\sum_{n=0}^{\infty} \sum_{k=0}^{n} c_{n-k} q_{k}(x) t^{n}
$$

On replacing $n$ by $n+k$, this becomes

$$
\begin{aligned}
\sum_{n=0}^{\infty} p_{n}(x) t^{n} & =\sum_{n=0}^{\infty} \sum_{k=0}^{\infty} c_{n} q_{k}(x) t^{n+k} \\
& =\sum_{k=0}^{\infty} q_{k}(x) t^{k} \sum_{n=0}^{\infty} c_{n} t^{n} .
\end{aligned}
$$

Setting $c_{n}=a_{n}^{(i)}(i$ is independent of $n$, where $i=1,2, \ldots, r)$, this becomes

$$
\begin{aligned}
\sum_{n=0}^{\infty} p_{n}(x) t^{n} & =\sum_{k=0}^{\infty} q_{k}(x) t^{k} \sum_{n=0}^{\infty} a_{n}^{(i)} t^{n}, \quad \text { by using Equation (2), we get } \\
& =\sum_{i=1}^{r} A_{i}(t)_{0} F_{q}\left(-; b_{1}, b_{2}, \ldots, b_{q} ; x H\left(\varepsilon_{i} t\right)\right) .
\end{aligned}
$$

This completes the proof.

Theorem 2 A necessary and sufficient condition that $p_{n}(x)$ be of $\sigma$-type zero and there exist a sequence $h_{k}$, independent of $x$ and $n$, such that

$$
\sum_{i=1}^{r} \varepsilon_{i}^{n} h_{n-1} \psi\left(\varepsilon_{i} t\right)=\sigma p_{n}(x)
$$

where $\psi\left(\varepsilon_{i} t\right)=A_{i}(t)_{0} F_{q}\left(-; b_{1}, b_{2}, \ldots, b_{q} ; x H\left(\varepsilon_{i} t\right)\right)$.

Proof If $p_{n}(x)$ is of $\sigma$-type zero, then it follows from Theorem 1 that

$$
\sum_{n=0}^{\infty} p_{n}(x) t^{n}=\sum_{i=1}^{r} A_{i}(t)_{0} F_{q}\left(-; b_{1}, b_{2}, \ldots, b_{q} ; x H\left(\varepsilon_{i} t\right)\right) .
$$

This can be written as

$$
\begin{aligned}
\sum_{n=0}^{\infty} \sigma p_{n}(x) t^{n} & =\sum_{i=1}^{r} A_{i}(t) \sigma_{0} F_{q}\left(-; b_{1}, b_{2}, \ldots, b_{q} ; x H\left(\varepsilon_{i} t\right)\right) \\
& =\sum_{i=1}^{r} H\left(\varepsilon_{i} t\right) A_{i}(t)_{0} F_{q}\left(-; b_{1}, b_{2}, \ldots, b_{q} ; x H\left(\varepsilon_{i} t\right)\right) \\
& =\sum_{i=1}^{r}\left(\sum_{n=0}^{\infty} h_{n} \varepsilon_{i}^{n+1} t^{n+1}\right) A_{i}(t)_{0} F_{q}\left(-; b_{1}, b_{2}, \ldots, b_{q} ; x H\left(\varepsilon_{i} t\right)\right) \\
& =\sum_{n=1}^{\infty} \sum_{i=1}^{r}\left(\varepsilon_{i}^{n} h_{n-1}\right) \psi\left(\varepsilon_{i} t\right) t^{n} .
\end{aligned}
$$


Thus

$$
\sigma p_{n}(x)=\sum_{i=1}^{r} \varepsilon_{i}^{n} h_{n-1} \psi\left(\varepsilon_{i} t\right)
$$

This completes the proof.

\section{Sheffer polynomials in two variables [12]}

Let $p_{n}(x, y)$ be of $\sigma$-type zero. Then $p_{n}(x, y)$ belongs to an operator $J(\sigma)=\sum_{k=0}^{\infty} c_{k} \sigma^{k+1}$, in which $c_{k}$ are constants and $c_{0} \neq 0$.

Since

$$
J(\sigma) p_{n}(x, y)=p_{n-1}(x, y), \quad n \geq 1,
$$

where

$$
\begin{array}{ll}
D_{x}=\frac{\partial}{\partial x}, \quad D_{y}=\frac{\partial}{\partial y}, & \theta=x \frac{\partial}{\partial x}, \quad \phi=y \frac{\partial}{\partial y}, \\
\sigma_{x}=D_{x} \prod_{m=1}^{p}\left(\theta+b_{m}-1\right), & \sigma_{y}=D_{y} \prod_{s=1}^{q}\left(\theta+b_{s}-1\right),
\end{array}
$$

and

$$
J((G+H)(t))=((G+H) J(t))=t, \quad \sigma=\sigma_{x}+\sigma_{y} .
$$

Theorem 3 A necessary and sufficient condition that $p_{n}(x, y)$ be of $\sigma$-type zero, with

$$
\sigma_{x}=D_{x} \prod_{m=1}^{p}\left(\theta+b_{m}-1\right), \quad \sigma_{y}=D_{y} \prod_{s=1}^{q}\left(\theta+b_{s}-1\right), \quad \sigma=\sigma_{x}+\sigma_{y},
$$

is that $p_{n}(x, y)$ possess a generating function in

$$
\sum_{i=1}^{r} A_{i}(t)_{0} F_{p}\left(-; b_{1}, b_{2}, \ldots, b_{p} ; x G\left(\varepsilon_{i} t\right)\right)_{0} F_{q}\left(-; c_{1}, c_{2}, \ldots, c_{q} ; y H\left(\varepsilon_{i} t\right)\right)=\sum_{n=0}^{\infty} p_{n}(x, y) t^{n}
$$

in which

$$
\begin{aligned}
& G(t)=\sum_{n=0}^{\infty} g_{n} t^{n+1}, \quad g_{0} \neq 0, \\
& H(t)=\sum_{n=0}^{\infty} h_{n} t^{n+1}, \quad h_{0} \neq 0, \\
& A_{i}(t)=\sum_{n=0}^{\infty} a_{n}^{(i)} t^{n} \quad\left(\text { not all } a_{0}^{(i)} \text { are zero }\right)
\end{aligned}
$$

and $i$ is independent of $n$. 
Proof Let $u_{i}={ }_{0} F_{p}\left(-; b_{1}, b_{2}, \ldots, b_{p} ; z_{i}\right)$ and $v_{i}={ }_{0} F_{q}\left(-; c_{1}, c_{2}, \ldots, c_{q} ; w_{i}\right)$ be the solutions of the following differential equations:

$$
\left[\theta_{z} \prod_{m=1}^{p}\left(\theta_{z}+b_{m}-1\right)-z_{i}\right] u_{i}=0, \quad \theta_{z}=z \frac{\partial}{\partial z}
$$

and

$$
\left[\phi_{w} \prod_{s=1}^{q}\left(\phi_{w}+c_{s}-1\right)-z_{i}\right] w_{i}=0, \quad \phi_{w}=w \frac{\partial}{\partial w} .
$$

On substituting $z_{i}=x G\left(\varepsilon_{i} t\right), w_{i}=y H\left(\varepsilon_{i} t\right)$ and keeping $t$ as a constant, where $\theta=x \frac{\partial}{\partial x}=\theta_{z}$, $\phi=y \frac{\partial}{\partial y}=\phi_{w}$, we get

$$
\theta \prod_{m=1}^{p}\left(\theta+b_{m}-1\right) u_{i}=x G\left(\varepsilon_{i} t\right) u_{i}
$$

and

$$
\phi \prod_{s=1}^{q}\left(\phi+c_{s}-1\right) w_{i}=y H\left(\varepsilon_{i} t\right) w_{i}
$$

This can also be written as

$$
\begin{aligned}
& \sigma_{0} F_{p}\left(-; b_{1}, b_{2}, \ldots, b_{p} ; x G\left(\varepsilon_{i} t\right)\right)_{0} F_{q}\left(-; c_{1}, c_{2}, \ldots, c_{q} ; y H\left(\varepsilon_{i} t\right)\right) \\
& \quad=\left\{G\left(\varepsilon_{i} t\right)+H\left(\varepsilon_{i} t\right)\right\}_{0} F_{p}\left(-; b_{1}, b_{2}, \ldots, b_{p} ; x G\left(\varepsilon_{i} t\right)\right)_{0} F_{q}\left(-; c_{1}, c_{2}, \ldots, c_{q} ; y H\left(\varepsilon_{i} t\right)\right) .
\end{aligned}
$$

Operating $J(\sigma)$ on both sides of Equation (4) yields

$$
\begin{aligned}
J(\sigma) & \sum_{n=0}^{\infty} p_{n}(x, y) t^{n} \\
= & J(\sigma) \sum_{i=1}^{r} A_{i}(t)_{0} F_{p}\left(-; b_{1}, b_{2}, \ldots, b_{p} ; x G\left(\varepsilon_{i} t\right)\right)_{0} F_{q}\left(-; c_{1}, c_{2}, \ldots, c_{q} ; y H\left(\varepsilon_{i} t\right)\right) \\
= & \sum_{i=1}^{r} A_{i}(t) J\left((G+H)\left(\varepsilon_{i} t\right)\right)_{0} F_{p}\left[-; b_{1}, b_{2}, \ldots, b_{p} ; x G\left(\varepsilon_{i} t\right)\right]_{0} F_{q}\left[-; c_{1}, c_{2}, \ldots, c_{q} ; y H\left(\varepsilon_{i} t\right)\right] \\
= & t \sum_{n=0}^{\infty} p_{n}(x, y) t^{n} \\
= & \sum_{n=1}^{\infty} p_{n-1}(x, y) t^{n} .
\end{aligned}
$$

Therefore, $J(\sigma) p_{0}(x, y)=0$ and $J(\sigma) p_{n}(x, y)=p_{n-1}(x, y), n \geq 1$.

Since $J(\sigma)$ is independent of $x$ and $y$, thus we arrive at the conclusion that $p_{n}(x, y)$ is of $\sigma$-type zero. 
Conversely, suppose $p_{n}(x, y)$ is of $\sigma$-type zero and belongs to the operator $J(\sigma)$. Now $q_{n}(x, y)$ is a simple set of polynomials. We can write

$$
\sum_{i=1}^{r}{ }_{0} F_{p}\left(-; b_{1}, b_{2}, \ldots, b_{p} ; x G\left(\varepsilon_{i} t\right)\right)_{0} F_{q}\left(-; c_{1}, c_{2}, \ldots, c_{q} ; y H\left(\varepsilon_{i} t\right)\right)=\sum_{n=0}^{\infty} p_{n}(x, y) t^{n} .
$$

Since $q_{n}(x, y)$ is a simple set, there exists a sequence $c_{k}$, independent of $n$, such that

$$
p_{n}(x, y)=\sum_{k=0}^{n} c_{n-k} q_{k}(x, y)
$$

and

$$
\sum_{n=0}^{\infty} p_{n}(x, y) t^{n}=\sum_{n=0}^{\infty} \sum_{k=0}^{n} c_{n-k} q_{k}(x, y) t^{n}
$$

On replacing $n$ by $n+k$, this becomes

$$
\begin{aligned}
& =\sum_{n=0}^{\infty} \sum_{k=0}^{\infty} c_{n} q_{k}(x, y) t^{n+k} \\
& =\sum_{k=0}^{\infty} q_{k}(x, y) t^{k} \sum_{n=0}^{\infty} c_{n} t^{n} .
\end{aligned}
$$

Setting $c_{n}=a_{n}^{(i)}(i$ is independent of $n$, where $i=1,2, \ldots, r)$, this becomes

$$
\begin{aligned}
& =\sum_{k=0}^{\infty} q_{k}(x, y) t^{k} \sum_{n=0}^{\infty} a_{n}^{(i)} t^{n} \\
& =\sum_{i=1}^{r} A_{i}(t)_{0} F_{p}\left(-; b_{1}, b_{2}, \ldots, b_{p} ; x G\left(\varepsilon_{i} t\right)\right)_{0} F_{q}\left(-; c_{1}, c_{2}, \ldots, c_{q} ; y H\left(\varepsilon_{i} t\right)\right) .
\end{aligned}
$$

This completes the proof.

Theorem 4 A necessary and sufficient condition that $p_{n}(x, y)$ be of $\sigma$-type zero and there exist sequences $g_{k}$ and $h_{k}$, independent of $x, y$ and $n$, such that

$$
\sum_{i=1}^{r} \varepsilon_{i}^{n}\left(g_{n-1}+h_{n-1}\right) v\left(\varepsilon_{i} t\right)=\sigma p_{n}(x, y)
$$

where $v\left(\varepsilon_{i} t\right)=A_{i}(t)_{0} F_{p}\left(-; b_{1}, b_{2}, \ldots, b_{p} ; x G\left(\varepsilon_{i} t\right)\right)_{0} F_{q}\left(-; c_{1}, c_{2}, \ldots, c_{q} ; y H\left(\varepsilon_{i} t\right)\right)$.

Proof If $p_{n}(x, y)$ is of $\sigma$-type zero, then it follows from Theorem 3 that

$$
\sum_{n=0}^{\infty} p_{n}(x, y) t^{n}=\sum_{i=1}^{r} A_{i}(t)_{0} F_{p}\left(-; b_{1}, b_{2}, \ldots, b_{p} ; x G\left(\varepsilon_{i} t\right)\right)_{0} F_{q}\left(-; c_{1}, c_{2}, \ldots, c_{q} ; y H\left(\varepsilon_{i} t\right)\right) .
$$


This can be written as

$$
\begin{aligned}
\sum_{n=0}^{\infty} \sigma p_{n}(x, y) t^{n}= & \sum_{i=1}^{r} A_{i}(t) \sigma_{0} F_{p}\left(-; b_{1}, b_{2}, \ldots, b_{p} ; x G\left(\varepsilon_{i} t\right)\right)_{0} F_{q}\left(-; c_{1}, c_{2}, \ldots, c_{q} ; y H\left(\varepsilon_{i} t\right)\right) \\
= & \sum_{i=1}^{r}(G+H)\left(\varepsilon_{i} t\right) A_{i}(t)_{0} F_{p}\left(-; b_{1}, b_{2}, \ldots, b_{p} ; x G\left(\varepsilon_{i} t\right)\right) \\
& \times{ }_{0} F_{q}\left(-; c_{1}, c_{2}, \ldots, c_{q} ; y H\left(\varepsilon_{i} t\right)\right) \\
= & \sum_{i=1}^{r}\left(\sum_{n=0}^{\infty}\left(g_{n}+h_{n}\right) \varepsilon_{i}^{n+1} t^{n+1}\right) A_{i}(t)_{0} F_{p}\left(-; b_{1}, b_{2}, \ldots, b_{p} ; x G\left(\varepsilon_{i} t\right)\right) \\
& \times{ }_{0} F_{q}\left(-; c_{1}, c_{2}, \ldots, c_{q} ; y H\left(\varepsilon_{i} t\right)\right) \\
= & \sum_{n=1}^{\infty} \sum_{i=1}^{r}\left(\varepsilon_{i}^{n}\left(g_{n-1}+h_{n-1}\right)\right) v\left(\varepsilon_{i} t\right) t^{n} .
\end{aligned}
$$

Thus

$$
\sigma p_{n}(x, y)=\sum_{i=1}^{r} \varepsilon_{i}^{n}\left(g_{n-1}+h_{n-1}\right) v\left(\varepsilon_{i} t\right)
$$

where $v\left(\varepsilon_{i} t\right)=A_{i}(t)_{0} F_{p}\left(-; b_{1}, b_{2}, \ldots, b_{p} ; x G\left(\varepsilon_{i} t\right)\right)_{0} F_{q}\left(-; c_{1}, c_{2}, \ldots, c_{q} ; y H\left(\varepsilon_{i} t\right)\right)$. This completes the proof.

\section{Competing interests}

The authors declare that they have no competing interests.

\section{Authors' contributions}

The authors contributed equally and significantly in writing this article. The authors read and approved the final manuscript.

\section{Acknowledgements}

Dedicated to Professor Hari M Srivastava.

The authors are indebted to the referees for their valuable suggestions which led to a better presentation of paper.

Received: 29 January 2013 Accepted: 19 April 2013 Published: 14 May 2013

\section{References}

1. Thorne, CJ: A property of Appell sets. Am. Math. Mon. 52, 191-193 (1945)

2. Srivastava, HM, Manocha, HL: A Treatise on Generating Functions. Wiley, New York (1984)

3. Dattoli, G, Migliorati, M, Srivastava, HM: Sheffer polynomials, monomiality principle, algebraic methods and the theory of classical polynomials. Math. Comput. Model. 45(9-10), 1033-1041 (2007)

4. Pintér, Á, Srivastava, HM: Addition theorems for the Appell polynomials and the associated classes of polynomial expansions. Aequ. Math. (2012). doi:10.1007/s00010-012-0148-8

5. Srivastava, HM, Choi, J: Zeta and q-Zeta Functions and Associated Series and Integrals. Elsevier, Amsterdam (2012)

6. Galiffa, JD: The Sheffer B-type 1 orthogonal polynomial sequences. Ph.D. Dissertation, University of Central Florida (2009)

7. Goldberg, JL: On the Sheffer A-type of polynomials generated by $A(t) \psi(x B(t))$. Proc. Am. Math. Soc. 17, 170-173 (1966)

8. Sheffer, IM: Note on Appell polynomials. Bull. Am. Math. Soc. 51, 739-744 (1945)

9. Rainville, ED: Special Functions. Macmillan Co., New York (1960)

10. Al Salam, WA, Verma, A: Generalized Sheffer polynomials. Duke Math. J. 37, 361-365 (1970)

11. Mc Bride, EB: Obtaining Generating Functions. Springer, New York (1971)

12. Alidad, B: On some problems of special functions and structural matrix analysis. Ph.D. Dissertation, Aligarh Muslim University (2008)

Cite this article as: Shukla et al.: On $\sigma$-type zero of Sheffer polynomials. Journal of Inequalities and Applications 2013 2013:241 